\title{
Informing robust decisions from workplace-based assessment
}

\begin{abstract}
Wilkinson, $T$.
New Zealand

Workplace based assessment have high validity but there is concern that robust high stakes decisions can only be made on the basis of formal high-stakes examinations. Drawing on experience of programmatic assessment and issues round failure to fail, this presentation will focus on how robust high stakes decisions can be made on the basis of workplace-based assessments alone. individual differences. Having several months of experience in delivering medical education through digital platforms has made us realize the need to sustain or do better to ensure the inclusivity and diversity of medical education in the new norm. There are many challenges faced by us to provide the best quality of medical education to learners. 'Think Global, Act Local' is a concept that considers enablers and barriers in the local setting, and charting strategies based on the best practices around the globe. 'Think Global, Act Local' can be a way to ensure medical education becoming more inclusive and more prepared to embrace diversity in the new norm.
\end{abstract}

